Brit. Heart J., 1963, 25, 784.

\title{
THE ELECTROCARDIOGRAM DURING THE FIRST WEEK OF LIFE*
}

\author{
BY \\ S. ZOE WALSH \\ From the Department of Padiatrics, Karolinska Hospital, and Wenner-Gren Cardiovascular Research Laboratory, \\ Norrtull's Hospital, Stockholm 60, Sweden \\ Received March 18, 1963
}

Most studies of the electrocardiogram in healthy full-term infants have commented on the presence of right axis deviation, a tendency to right ventricular preponderance, and changes in amplitude and direction of præcordial T waves (Krumbhaar and Jenks, 1917; Schaffer et al.,1950; Ziegler, 1951, 1956). Surprisingly few serial studies have been done, despite increased interest in the neonatal period and the importance of determining individual as well as group differences (Datey and Bharucha, 1960; Michaëlsson, 1959). Recent investigations have demonstrated significant changes in electrocardiographic intervals during the first hour as well as the first week of life (Walsh, 1963a, c). It was therefore thought to be of interest to determine whether the amplitude of the QRS complex was similarly affected.

\section{SubJECTS AND METHODS}

Sixteen-lead electrocardiograms, including standard extremity and unipolar præcordial leads V1 to V6, V4R, Ve, $2 \mathrm{~V} 1$, and $2 \mathrm{~V} 2 \dagger$, were taken on 100 healthy full-term infants on the first, second, third, and fifth or sixth days of life. Three of them were subsequently excluded because of complete right bundle-branch block, Wolff-Parkinson-White syndrome (Walsh, 1963d), and wandering pacemaker. Of the remaining 97, 4 were not examined on their first day of life. In no instance was there clinical evidence of heart disease on the basis of auscultation or radiological findings. All were well at the time of examination, and maternal anæsthesia had been minimal or had not been given.

In the determination of the effect of age on the amplitude of deflections, analysis of means and distribution of values were confined to 68 infants subjected to all 4 examinations, 42 of whom were female. These infants were divided into 4 groups, according to the time after birth of the initial examination:

Group Ia-30 minutes old or less (17 infants)

Group Ib-35 minutes to 1 hour old (21 infants)

Group IIa-65 minutes to 4 hours old (13 infants)

Group IIb $-4 \frac{1}{4}$ to 18 hours old (17 infants).

When there were no differences, the groups were combined for investigation of differences at various ages. Data on the remaining 29 infants were included in the tables when minimum and maximum values were exceeded. Individual differences from the first to subsequent examinations were also calculated.

All electrocardiograms were taken by the author, with the aid of an assistant, employing a four-channel jet writer at a paper speed of $100 \mathrm{~mm}$./sec. Accuracy was enhanced by using an external voltmeter and

* This work was done during the tenure of an Advanced Research Fellowship of the American Heart Association and supported by grants from the American Heart Association and the International Society of Cardiology Foundation.

$\dagger$ Leads Ve, 2V1, and $2 \mathrm{~V} 2$ were taken at the tip of the xyphoid process and the second right and left intercostal spaces respectively. 
periodically testing the speed. All leads were recorded at normal standardization $(1 \mathrm{mV}=1 \mathrm{~cm}$. $)$ and measurements made with a magnifying lens of $5 \times$. The smallest unit of measurement was $0.5 \mathrm{~mm}$. $(0.05 \mathrm{mV})$. The extremity electrodes measured $3.5 \times 2.0 \mathrm{~cm}$, and the circular chest electrodes had a diameter of $1.3 \mathrm{~cm}$. Great care was exercised in selecting electrode positions and in applying and removing electrode paste. No sedation was given. The infants were supine and asleep or resting quietly at the time of examination.

\section{RESULTS}

$Q$ Wave. A $\mathrm{Q}$ wave was present in all leads except $2 \mathrm{~V} 1$ and $2 \mathrm{~V} 2$ at some time during the first week of life. In lead V4R, 9 infants had a $Q$ wave with maximum amplitude of $1.5 \mathrm{~mm} .(0.15 \mathrm{mV})$ on initial examination: in 4 of them, a $Q$ wave was present in V6. On the second day, 2 of 12 infants with a $Q$ wave had this wave in V6 also. By the end of the week, all 4 infants with a persistent $\mathrm{Q}$ wave had had this finding previously. A single instance of a $\mathrm{Q}$ wave was seen in V1 and V2 in 2 different infants on their fourth examination: both of them had $\mathrm{Q}$ waves in Ve and V4R also. Fourteen infants had a $\mathrm{Q}$ wave at some time during the first week of life in lead Ve, which never exceeded $1.5 \mathrm{~mm}$. $(0.15 \mathrm{mV})$ in magnitude. Of these, 9 infants had this wave on at least two examinations, and 4 on all four examinations. Age did not appear to influence the incidence of this deflection. Of a total of $34 \mathrm{Q}$ waves in this lead at any age, 20 had an associated $\mathrm{Q}$ wave in V4R.

Although no $\mathrm{Q}$ waves were present in V3 initially, 5 infants had this finding by the end of the week. In V4, V5, and V6, the incidence increased with age, and by the last examination, only 14 of 68 infants did not have a $\mathrm{Q}$ wave in V6. All of these increases with age were significant (V4-p $<0.05 ; \mathrm{V} 5-\mathrm{p}<0.001 ; \mathrm{V} 6-\mathrm{p}<0.01)$. Though the incidence increased with age, it is noteworthy that on initial examination only 3 of 93 infants had no $Q$ waves in any of the extremity or præcordial leads. None subsequently had this finding, apart from 1 infant in whom the absence persisted until the age of 47 hours, when he was lost to follow-up.

According to Groedel and Miller (1944), a Q wave in lead I is present in the newborn infant only when there is a $Q$ wave in left præcordial leads. With the exception of 2 infants, one of whom had this finding on three examinations, this was confirmed. A $Q$ wave was recorded in V4R, Ve, and V1 in 2 normal infants aged 2 and 4 days by Ziegler (1951); one of them had a Q wave in left præcordial leads. The only other infant with this finding in his series of 600 infants and children was $5 \frac{1}{2}$ months old. According to Rosen and Gardberg (1957), Q waves in V4R and V1 are exceptional at birth but may be present at 1 month and can persist throughout infancy. However, none of the infants studied was less than 24 hours of age, and all infants more than 1 day and less than 1 month old constituted one group. In another study (Rothfeld et al., 1960), of 50 healthy newborns singly examined one had this wave in lead V1. The findings of these investigators, in conjunction with those described here, suggest that contrary to a number of reports $Q$ waves in these leads are an uncommon but normal variant during early infancy. Furthermore, it seems likely that more complete exploration of the right chest would reveal a higher incidence of $Q$ waves in these leads. As no $\mathrm{Q}$ waves were registered in leads $2 \mathrm{~V} 1$ or $2 \mathrm{~V} 2$ during the first week of life in any of the 97 full-term infants, nor in another study of 82 healthy premature infants serially examined during the first year of life (Walsh, 1963b), these leads may merit further investigation.

$R$ Wave. On the first day of life, significantly higher mean values, primarily over the midpræcordium, were registered in Group I infants, i.e., those less than 1 hour old. These differences were statistically significant in leads V2 (p<0.05), V3 (p<0.01), V4 $(p<0.05)$, and were just short of significance in V1, V5, and V6. However, on determining the distribution of values in V1, 12 infants in Group I were found to have an R wave amplitude of $24 \mathrm{~mm}$. or more $(2.4 \mathrm{mV})$ while only 2 infants in Group II (those over 1 hour old on initial examination) were in this range. This difference was significant $(\mathrm{p}<0.05)$. Although differences were just short of significant in V5 and V6, and not significant in $\mathrm{V} 4 \mathrm{R}, \mathrm{Ve}, 2 \mathrm{~V} 1$, and $2 \mathrm{~V} 2$, the trend was generally in the same direction with higher mean, minimum, and maximum values in Group I infants. These differences were of slightly greater significance when infants in Group Ia were compared with those in Group IIb (Fig. 1 and 2). 
This trend was further demonstrated when values were compared on the first and last examination. Significant decreases in amplitude of the $\mathrm{R}$ wave were observed primarily in right præcordial leads in Group I infants (leads V1-p $<0.05$; V2, Ve-p $<0.001$; and V $3-p<0.01$ ). The decreases in leads V4R ( $\mathrm{p}<0.01)$ and $2 \mathrm{~V} 2(\mathrm{p}<0.05)$ were also found to be significant, and the decrease in lead 2V1 was just short of significance in infants in Group Ia. A similar but less obvious decrease in amplitude was noted in lead Ve in Group II infants $(\mathrm{p}<0.05)$ and in leads V4R and $2 \mathrm{~V} 1(\mathrm{p}<0.05)$ in Group IIb infants. Significant increases over the left præcordium were recorded only in the latter group (leads V4, V6-p $<0.05 ;$ V $5-\mathrm{p}<0.01$ ) (Table I). When mean individual differences were calculated, decreases were observed in all præcordial leads of infants in Group I. Less notable decreases were found in Group II infants, but only in right præcordial leads, while increases occurred in leads V3-V6. As higher amplitudes were present in the youngest infants on the first day of life, the disparity between the groups seems readily explained.

In a previous report, significant differences in $\mathrm{Q}-\mathrm{R}$ time (or onset of the intrinsicoid deflection) in V1 and V6 were noted during the first week of life (Walsh, 1963c). It was thought to be of interest to relate this interval to the amplitude of the respective $R$ wave. There appeared to be a positive correlation between amplitude of the $\mathbf{R}$ wave and duration of this interval, especially in lead V6, i.e., higher amplitude waves tended to be associated with longer intervals. These differences were statistically significant in lead V1 on the first day of life in Group I infants. In the class 0.01$0.015 \mathrm{sec}$. Q-R time, the mean $\mathrm{R}$ wave amplitude was $17.9 \mathrm{~mm}$. $(1.78 \mathrm{mV})$, while in the class

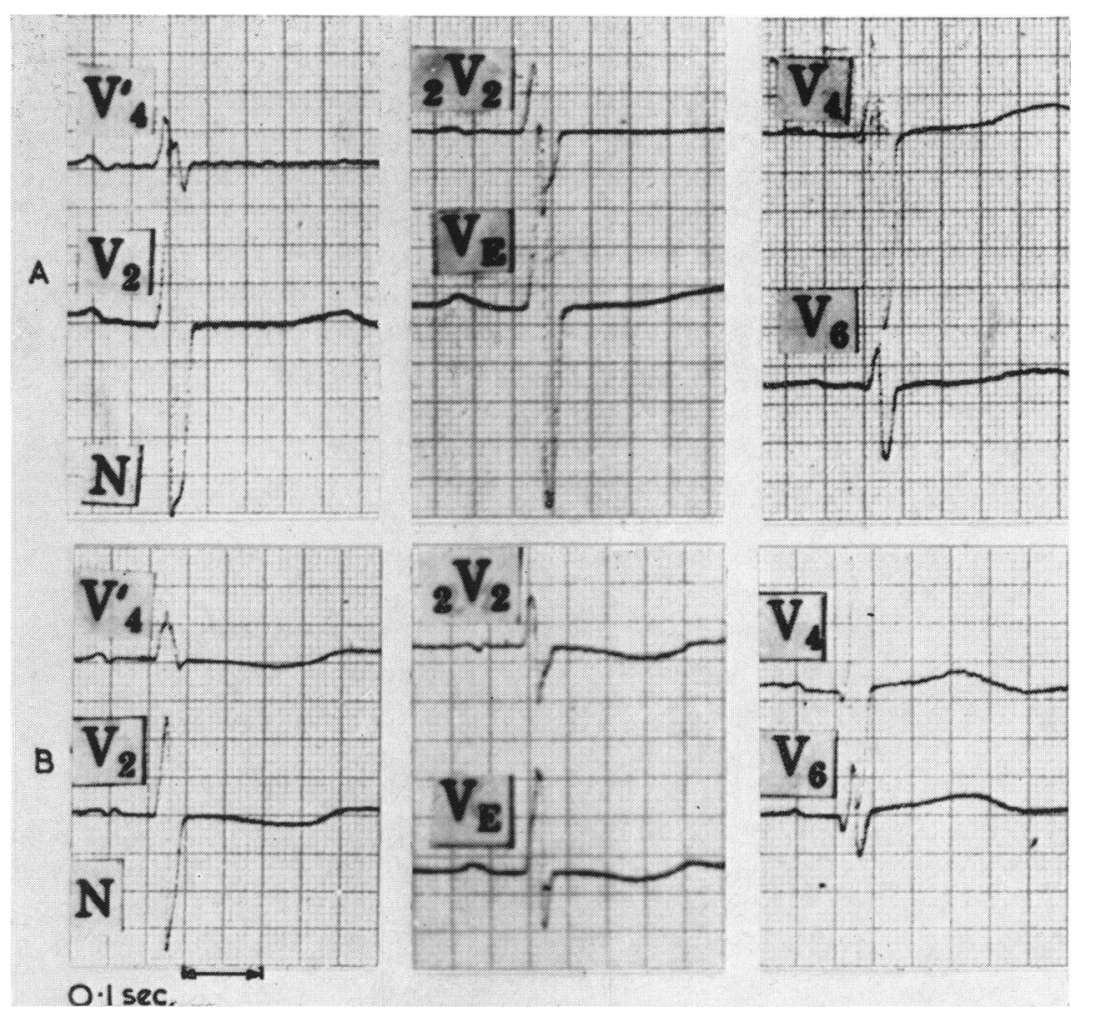

FIG. 1.-Female infant (Group Ia). Tracings recorded at 30 minutes (A) and at 6 days (B). Note initial high amplitude QRS deflections and absence of $Q$ waves in V4 and V6. (N=normal standardization; $V^{\prime} 4=V 4 R$; distance between fine horizontal lines, $1 \mathrm{~mm}$.). 


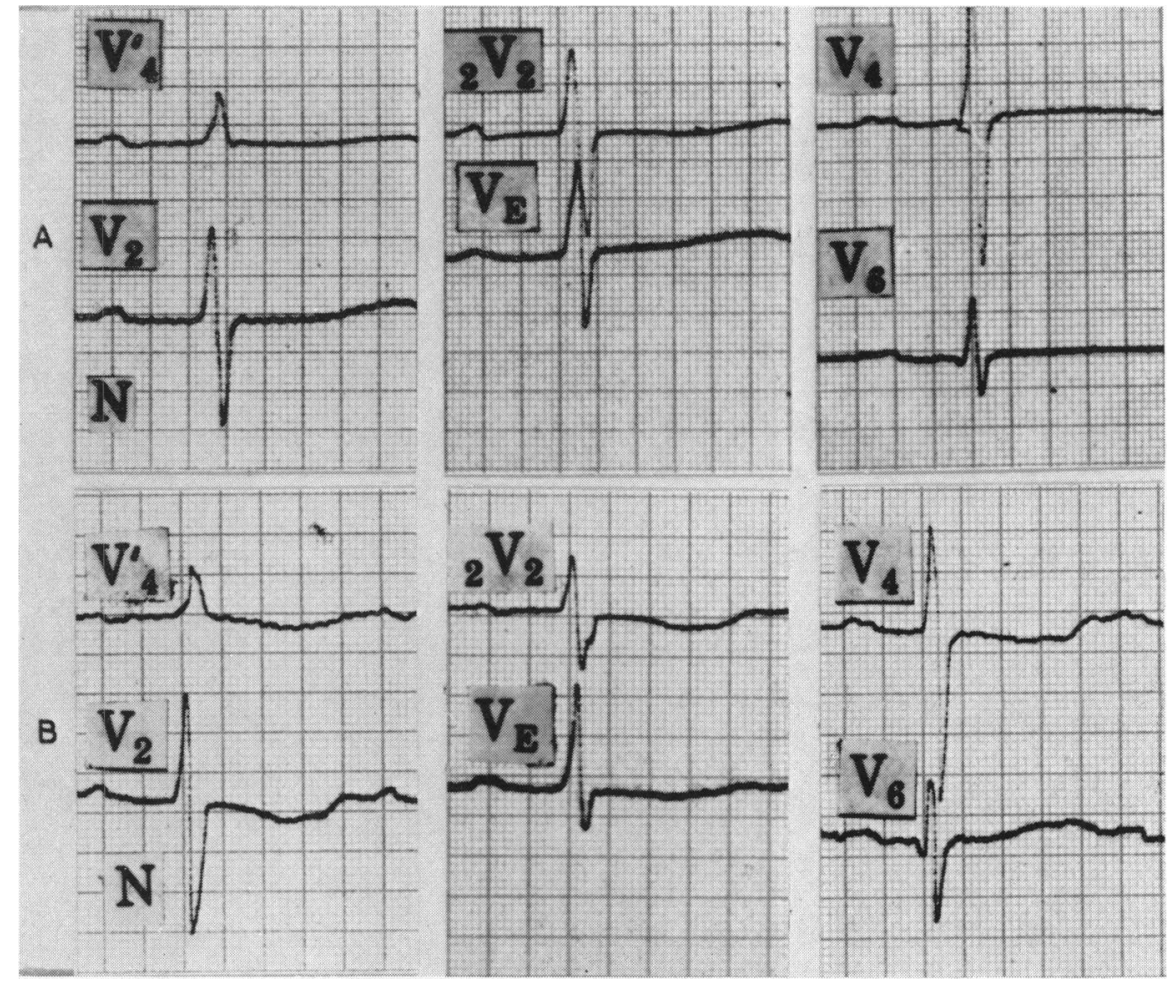

Fig. 2.-Female infant (Group IIb). Tracings recorded at $7 \frac{1}{2}$ hours (A) and at 6 days (B). Relatively lower amplitude QRS deflections and small Q wave in lead V6 are present on initial examination. A less noteworthy decrease in magnitude of deflections occurs with age.

TABLE I

R Wave Amplitude in 1/10 mV showing Minimum, Mean, Maximum and Standard Deviation DURING THE FIRST WEFK OF LIFE

\begin{tabular}{|c|c|c|c|c|c|c|c|c|c|c|c|}
\hline \multirow{2}{*}{ Lead } & \multirow{2}{*}{ Day } & \multicolumn{5}{|c|}{ Group I } & \multicolumn{5}{|c|}{ Group II } \\
\hline & & No. & Min. & Mean & Max. & S.D. & No. & Min. & Mean & Max. & S.D. \\
\hline \multirow{3}{*}{ V4R } & 1 & 38 & $\begin{array}{c}(3 \cdot 0) \\
5 \cdot 0 \\
(2 \cdot 5)\end{array}$ & $11 \cdot 6$ & 20.0 & 3.9 & 28 & $\begin{array}{c}(3.5) \\
5.0\end{array}$ & & $\begin{array}{c}(18 \cdot 0) \\
17 \cdot 0 \\
(19.0)\end{array}$ & $5 \cdot 8$ \\
\hline & $\begin{array}{l}2 \\
3\end{array}$ & $\begin{array}{l}38 \\
37\end{array}$ & $\begin{array}{l}3.0 \\
2.5\end{array}$ & $\begin{array}{r}10 \cdot 1 \\
9.9\end{array}$ & $\begin{array}{l}20.0 \\
20 \cdot 0\end{array}$ & $\begin{array}{l}4 \cdot 7 \\
3 \cdot 8\end{array}$ & $\begin{array}{l}28 \\
30\end{array}$ & $\begin{array}{l}4 \cdot 0 \\
5 \cdot 0\end{array}$ & $\begin{array}{l}10.40 \\
10.55\end{array}$ & $\begin{array}{r}16.5 \\
22.0\end{array}$ & $\begin{array}{l}5 \cdot 0 \\
5 \cdot 1\end{array}$ \\
\hline & $5-6$ & 38 & $5 \cdot 5$ & $10 \cdot 1$ & $18 \cdot 0$ & $3 \cdot 4$ & 30 & 5.0 & $9 \cdot 60$ & 16.5 & $5 \cdot 7$ \\
\hline \multirow{3}{*}{ V1 } & 1 & 38 & $\begin{array}{c}(7 \cdot 5) \\
9 \cdot 0\end{array}$ & $21 \cdot 0$ & 32.0 & $5 \cdot 1$ & 29 & $\begin{array}{c}(7.5) \\
10.0 \\
(8.5)\end{array}$ & $19 \cdot 10$ & $\begin{array}{r}26 \cdot 0 \\
(28 \cdot 5)\end{array}$ & $3 \cdot 7$ \\
\hline & $\begin{array}{l}2 \\
3\end{array}$ & $\begin{array}{l}37 \\
37\end{array}$ & $\begin{array}{l}8 \cdot 0 \\
9 \cdot 0\end{array}$ & $\begin{array}{l}20 \cdot 3 \\
19 \cdot 0\end{array}$ & $\begin{array}{l}30.0 \\
30.0\end{array}$ & $\begin{array}{l}5.0 \\
4.6\end{array}$ & $\begin{array}{l}30 \\
30\end{array}$ & $\begin{array}{l}9 \cdot 0 \\
7 \cdot 5\end{array}$ & $\begin{array}{l}19 \cdot 10 \\
19.80\end{array}$ & $\begin{array}{l}28 \cdot 0 \\
32 \cdot 0\end{array}$ & $\begin{array}{l}4.6 \\
5.0\end{array}$ \\
\hline & $5-6$ & 38 & $\begin{array}{c}(6.0) \\
9.0\end{array}$ & $18 \cdot 2$ & 29.0 & 4.9 & 30 & 7.0 & $18 \cdot 50$ & $\begin{array}{c}(33.0) \\
28.0\end{array}$ & 5.0 \\
\hline
\end{tabular}


TABLE I (continued)

\begin{tabular}{|c|c|c|c|c|c|c|c|c|c|c|c|}
\hline \multirow{2}{*}{ Lead } & \multirow{2}{*}{ Day } & \multicolumn{5}{|c|}{ Group I } & \multicolumn{5}{|c|}{ Group II } \\
\hline & & No. & Min. & Mean & Max. & S.D. & No. & Min. & Mean & Max. & S.D. \\
\hline V2 & $\begin{array}{c}1 \\
2 \\
3 \\
5-6\end{array}$ & $\begin{array}{l}38 \\
38 \\
38 \\
38\end{array}$ & $\begin{array}{c}(8 \cdot 0) \\
13 \cdot 0 \\
(8.0) \\
12.0 \\
11 \cdot 0 \\
5.5\end{array}$ & $\begin{array}{r}24 \cdot 2 \\
23 \cdot 2 \\
21 \cdot 9 \\
19 \cdot 6\end{array}$ & $\begin{array}{l}34 \cdot 0 \\
38 \cdot 0 \\
34 \cdot 0 \\
30 \cdot 0\end{array}$ & $\begin{array}{l}5 \cdot 2 \\
6 \cdot 7 \\
5 \cdot 1 \\
6 \cdot 0\end{array}$ & $\begin{array}{l}29 \\
29 \\
30 \\
30\end{array}$ & $\begin{array}{c}11.0 \\
(9.5) \\
10.0 \\
\\
10.0 \\
(10.5) \\
12.0\end{array}$ & $\begin{array}{l}21 \cdot 30 \\
21 \cdot 50 \\
19 \cdot 30 \\
20 \cdot 10\end{array}$ & $\begin{array}{c}32 \cdot 5 \\
36 \cdot 5 \\
(27 \cdot 0) \\
25 \cdot 5 \\
27 \cdot 5\end{array}$ & $\begin{array}{l}4 \cdot 1 \\
5 \cdot 7 \\
4 \cdot 0 \\
4 \cdot 0\end{array}$ \\
\hline V3 & $\begin{array}{c}1 \\
2 \\
3 \\
5-6\end{array}$ & $\begin{array}{l}38 \\
\\
37 \\
38 \\
\\
38\end{array}$ & $\begin{array}{c}9.0 \\
(7.5) \\
11.0 \\
8.0 \\
(8.0) \\
9.0\end{array}$ & $\begin{array}{l}21 \cdot 7 \\
19 \cdot 8 \\
20 \cdot 0 \\
18 \cdot 8\end{array}$ & $\begin{array}{l}30.0 \\
29.0 \\
30.0 \\
30.0\end{array}$ & $\begin{array}{l}4 \cdot 8 \\
4 \cdot 3 \\
4 \cdot 3 \\
4 \cdot 6\end{array}$ & $\begin{array}{l}29 \\
30 \\
30 \\
\\
30\end{array}$ & $\begin{array}{c}(9.0) \\
9.5 \\
(11.0) \\
12.0 \\
6.0 \\
(10.5) \\
12.0\end{array}$ & $\begin{array}{c}18 \cdot 70 \\
19 \cdot 60 \\
18 \cdot 90 \\
19 \cdot 50\end{array}$ & $\begin{array}{l}28 \cdot 0 \\
34 \cdot 0 \\
34 \cdot 0 \\
33 \cdot 0\end{array}$ & $\begin{array}{l}3 \cdot 7 \\
4 \cdot 4 \\
4 \cdot 5 \\
4 \cdot 1\end{array}$ \\
\hline V4 & $\begin{array}{c}1 \\
2 \\
3 \\
5-6\end{array}$ & $\begin{array}{l}38 \\
38 \\
\\
38 \\
\\
38\end{array}$ & $\begin{array}{c}(10.5) \\
11.0 \\
6.0 \\
(8.0) \\
10.0 \\
9.0\end{array}$ & $\begin{array}{r}19 \cdot 8 \\
16 \cdot 7 \\
17 \cdot 9 \\
19 \cdot 8\end{array}$ & $\begin{array}{r}33 \cdot 0 \\
29 \cdot 0 \\
33 \cdot 0 \\
32 \cdot 0\end{array}$ & $\begin{array}{l}6 \cdot 2 \\
5 \cdot 2 \\
5 \cdot 2 \\
5 \cdot 5\end{array}$ & $\begin{array}{l}29 \\
30 \\
\\
29 \\
\\
30\end{array}$ & $\begin{array}{c}(6 \cdot 0) \\
7.0 \\
5 \cdot 5 \\
(3 \cdot 5) \\
4.0 \\
\\
8.0\end{array}$ & $\begin{array}{r}16 \cdot 70 \\
17 \cdot 40 \\
\\
16 \cdot 60 \\
18 \cdot 90\end{array}$ & $\begin{array}{c}30.0 \\
34.5 \\
\\
33.0 \\
(31.0) \\
29.0\end{array}$ & $\begin{array}{l}5 \cdot 4 \\
6 \cdot 1 \\
5 \cdot 0 \\
5 \cdot 4\end{array}$ \\
\hline V5 & $\begin{array}{c}1 \\
2 \\
3 \\
5-6\end{array}$ & $\begin{array}{l}38 \\
38 \\
38 \\
38\end{array}$ & $\begin{array}{l}2 \cdot 5 \\
2 \cdot 5 \\
2 \cdot 5 \\
2 \cdot 5\end{array}$ & $\begin{array}{l}12 \cdot 2 \\
10 \cdot 4 \\
10 \cdot 2 \\
12 \cdot 0\end{array}$ & $\begin{array}{l}26 \cdot 0 \\
27 \cdot 0 \\
23 \cdot 5 \\
25 \cdot 0\end{array}$ & $\begin{array}{l}3 \cdot 3 \\
3 \cdot 4 \\
3 \cdot 9 \\
3 \cdot 1\end{array}$ & $\begin{array}{l}29 \\
30 \\
30 \\
30\end{array}$ & $\begin{array}{l}2 \cdot 0 \\
2 \cdot 5 \\
2 \cdot 5 \\
3 \cdot 0\end{array}$ & $\begin{array}{r}10 \cdot 50 \\
10 \cdot 80 \\
9 \cdot 25 \\
13 \cdot 20\end{array}$ & $\begin{array}{l}25 \cdot 0 \\
27 \cdot 0 \\
23 \cdot 5 \\
26 \cdot 0\end{array}$ & $\begin{array}{l}5 \cdot 3 \\
5 \cdot 5 \\
4 \cdot 5 \\
6 \cdot 2\end{array}$ \\
\hline V6 & $\begin{array}{c}1 \\
2 \\
3 \\
5-6\end{array}$ & $\begin{array}{l}38 \\
38 \\
38 \\
38\end{array}$ & $\begin{array}{l}1 \cdot 5 \\
0 \\
0 \cdot 5 \\
1 \cdot 0\end{array}$ & $\begin{array}{l}9 \cdot 2 \\
7 \cdot 2 \\
7 \cdot 5 \\
9 \cdot 4\end{array}$ & $\begin{array}{l}21 \cdot 0 \\
17 \cdot 5 \\
20 \cdot 5 \\
22 \cdot 0\end{array}$ & $\begin{array}{l}4 \cdot 9 \\
4 \cdot 4 \\
5 \cdot 1 \\
5 \cdot 3\end{array}$ & $\begin{array}{l}29 \\
30 \\
29 \\
30\end{array}$ & $\begin{array}{l}0 \\
0 \\
0 \\
1 \cdot 0\end{array}$ & $\begin{array}{l}7 \cdot 10 \\
6 \cdot 00 \\
6 \cdot 40 \\
9 \cdot 60\end{array}$ & $\begin{array}{l}19 \cdot 0 \\
20.0 \\
19.0 \\
27.0\end{array}$ & $\begin{array}{l}4 \cdot 7 \\
4 \cdot 7 \\
5 \cdot 0 \\
6 \cdot 0\end{array}$ \\
\hline $\mathrm{Ve}$ & $\begin{array}{c}1 \\
2 \\
3 \\
5-6\end{array}$ & $\begin{array}{l}38 \\
38 \\
38 \\
37\end{array}$ & $\begin{array}{l}16.0 \\
11.5 \\
10.5 \\
10.0\end{array}$ & $\begin{array}{l}24 \cdot 9 \\
22 \cdot 3 \\
22 \cdot 5 \\
18 \cdot 5\end{array}$ & $\begin{array}{l}37 \cdot 5 \\
37 \cdot 0 \\
34 \cdot 0 \\
32 \cdot 0\end{array}$ & $\begin{array}{l}5 \cdot 1 \\
5 \cdot 1 \\
4 \cdot 9 \\
5 \cdot 5\end{array}$ & $\begin{array}{l}29 \\
29 \\
30 \\
29\end{array}$ & $\begin{array}{r}(7 \cdot 5) \\
13.0 \\
12.0 \\
9.0 \\
11.5\end{array}$ & $\begin{array}{l}22 \cdot 95 \\
22 \cdot 40 \\
22 \cdot 60 \\
20 \cdot 20\end{array}$ & $\begin{array}{c}(35 \cdot 0) \\
34 \cdot 5 \\
31 \cdot 0 \\
32 \cdot 5 \\
32.0\end{array}$ & $\begin{array}{l}5 \cdot 4 \\
4 \cdot 3 \\
5 \cdot 4 \\
4 \cdot 7\end{array}$ \\
\hline 2V1 & $\begin{array}{c}1 \\
2 \\
3 \\
5-6\end{array}$ & $\begin{array}{l}38 \\
38 \\
\\
38 \\
\\
38\end{array}$ & $\begin{array}{c}(3 \cdot 5) \\
5 \cdot 5 \\
3 \cdot 5 \\
\\
3 \cdot 0 \\
3.0\end{array}$ & $\begin{array}{r}12.0 \\
11.0 \\
10.6 \\
9.5\end{array}$ & $\begin{array}{l}24 \cdot 0 \\
18 \cdot 0 \\
24 \cdot 0 \\
22 \cdot 0\end{array}$ & $\begin{array}{r}4 \cdot 1 \\
3 \cdot 8 \\
4 \cdot 5 \\
\\
4 \cdot 4\end{array}$ & $\begin{array}{l}29 \\
30 \\
\\
29 \\
30\end{array}$ & $\begin{array}{r}4 \cdot 0 \\
3 \cdot 0 \\
4 \cdot 0 \\
4 \cdot 0\end{array}$ & $\begin{array}{r}11.90 \\
10.80 \\
9.40 \\
9.60\end{array}$ & $\begin{array}{c}20.5 \\
25.0 \\
(19.0) \\
15.0 \\
(19.0) \\
14.0\end{array}$ & $\begin{array}{l}4 \cdot 7 \\
4 \cdot 4 \\
\\
2 \cdot 7 \\
\\
2 \cdot 4\end{array}$ \\
\hline $2 \mathrm{~V} 2$ & $\begin{array}{c}1 \\
2 \\
3 \\
5-6\end{array}$ & $\begin{array}{l}38 \\
\\
38 \\
38 \\
\\
37\end{array}$ & $\begin{array}{c}(4 \cdot 0) \\
5 \cdot 0 \\
\\
5 \cdot 0 \\
5 \cdot 0 \\
(3 \cdot 5) \\
6 \cdot 0\end{array}$ & $\begin{array}{l}14 \cdot 1 \\
13 \cdot 3 \\
13 \cdot 5 \\
10 \cdot 2\end{array}$ & $\begin{array}{l}28 \cdot 0 \\
23 \cdot 0 \\
28 \cdot 0 \\
27 \cdot 0\end{array}$ & $\begin{array}{l}5 \cdot 2 \\
4 \cdot 8 \\
5 \cdot 4 \\
5 \cdot 2\end{array}$ & $\begin{array}{l}29 \\
29 \\
30 \\
30\end{array}$ & $\begin{array}{c}(5 \cdot 5) \\
6 \cdot 0 \\
(6 \cdot 0) \\
7 \cdot 5 \\
4 \cdot 5 \\
4 \cdot 5\end{array}$ & $\begin{array}{l}13 \cdot 20 \\
12 \cdot 60 \\
11 \cdot 20 \\
11 \cdot 30\end{array}$ & $\begin{array}{l}25 \cdot 0 \\
20 \cdot 0 \\
20 \cdot 5 \\
20 \cdot 0\end{array}$ & $\begin{array}{l}4 \cdot 8 \\
3 \cdot 6 \\
4 \cdot 2 \\
3 \cdot 3\end{array}$ \\
\hline
\end{tabular}

On the first day of life values are generally greater in Group I infants. Figures in parentheses indicate minimum and maximum values in infants without all 4 examinations. Each zero corresponds to a single value. 
$0.02-0.025 \mathrm{sec}$, , the mean $\mathrm{R}$ wave amplitude was $22.2 \mathrm{~mm} .(2 \cdot 2 \mathrm{mV})(\mathrm{p}<0.05)$. In $\mathrm{V} 6$, significant differences were noted in both groups, on the first day of life in Group II infants and at the end of the week in Group I infants. In all instances, however, the same trend was maintained (Table II).

TABLE II

Relation between Duration of Q-R Time and Mean Amplitude of the R Wave in $1 / 10$ MV in Leads V1 and V6 ON First and FifTh or Sixth Day of Life

\begin{tabular}{|c|c|c|c|c|c|}
\hline \multirow{3}{*}{ Lead } & \multirow{3}{*}{ Group } & \multicolumn{2}{|c|}{ Day 1} & \multicolumn{2}{|c|}{ Day 5-6 } \\
\hline & & \multicolumn{4}{|c|}{$\mathrm{Q}-\mathrm{R}$ time (sec.) } \\
\hline & & $0.005-0.020$ & $0.025-0.035$ & $0.005-0.020$ & $0.025-0.035$ \\
\hline \multirow{2}{*}{ V1 } & $\mathbf{I}$ & $\begin{array}{l}20 \cdot 5 \\
(5 \cdot 8)\end{array}$ & $\begin{array}{c}22 \cdot 3 \\
(5 \cdot 3)\end{array}$ & $\begin{array}{l}17 \cdot 3 \\
(4 \cdot 9)\end{array}$ & $\begin{array}{c}21 \cdot 0 \\
(5 \cdot 2)\end{array}$ \\
\hline & II & $\begin{array}{l}18 \cdot 7 \\
(4 \cdot 5)\end{array}$ & $\begin{array}{l}20 \cdot 4 \\
(3 \cdot 6)\end{array}$ & $\begin{array}{l}17 \cdot 4 \\
(4 \cdot 6)\end{array}$ & $\begin{array}{l}20 \cdot 4 \\
(5 \cdot 3)\end{array}$ \\
\hline \multirow{3}{*}{ V6 } & I & $\begin{array}{c}9 \cdot 0 \\
(5 \cdot 1)\end{array}$ & $\begin{array}{c}9 \cdot 3 \\
(5 \cdot 0)\end{array}$ & $\begin{array}{c}7 \cdot 8 \\
(4 \cdot 2)\end{array}$ & $\begin{array}{c}12 \cdot 3 \\
(6 \cdot 1)\end{array}$ \\
\hline & & & & \multicolumn{2}{|c|}{$(p<0.01)$} \\
\hline & II & $\begin{array}{c}5 \cdot 5 \\
(3 \cdot 8)\end{array}$ & $\begin{array}{r}9 \cdot 4 \\
\\
55)\end{array}$ & $\begin{array}{c}8 \cdot 1 \\
(5 \cdot 9)\end{array}$ & $\begin{array}{l}10 \cdot 3 \\
(3 \cdot 9)\end{array}$ \\
\hline
\end{tabular}

Figures in parentheses are standard deviations. A trend towards a positive correlation is present in all instances.

$S$ Wave. On the first day, mean amplitudes over the left præcordium were greater in Group I infants. The difference was moderately significant in lead V6 $(\mathrm{p}<0.01)$ and just short of significance in leads V4 and V5 $(p<0.06)$. A comparison of values for infants in Group Ia with those in Group IIb showed the difference to be significant also in V4, and just short of significance in Ve. Larger amplitude $S$ waves were encountered in lead $2 \mathrm{~V} 2$ in the latter group $(\mathrm{p}<0.05)$ (Fig. 1 and 2$)$.

Decreases in mean amplitude of the $S$ wave from the first to the last examination occurred in almost all leads in the two main groups. These decreases were significant for Group I infants in leads from the right and mid-præcordium (leads V1-p $<0.05$; V2-p $<0.01$; V3, V4, and Ve$\mathrm{p}<0.001)$. The decreases were significant for Group Ia infants in leads from the left chest as well as just short of significance in lead V4R (leads V5-p $<0.01$; V6-p $<0.05$; V4R-p $<0.06$ ). The decreases were significant only in right chest leads for infants in Group IIb (leads V4R, V2, 2V1$\mathrm{p}<0.05$; V3, Ve-p $<0.001)$. However, when individual differences were calculated, a mean decrease occurred in all præcordial leads of all infants apart from lead V6 in Group II infants.

In lead V3, infants in Group II showed a significant increase in amplitude from the first to the second day of life $(p<0.05)$. A significant change from the first to the third day of life was also noted in this lead. Ten infants on initial examination had an $S$ wave of $10 \mathrm{~mm}$. or less $(1.0 \mathrm{mV})$, whereas none was in this range on the subsequent examination $(p<0.01)$.

From the third to the fifth or sixth day of life, highly significant decreases in amplitude of the $S$ wave occurred in leads V1, V2, and V3 $(\mathrm{p}<0.001)$. In V6, a significant decrease in incidence of minimum and maximum values was seen $(\mathrm{p}<0.05)$ (Table III).

The range and mean amplitudes of $R$ and $S$ waves accord with those of Ziegler (1951), but as he included infants from 1 to 7 days in a single group and did not state how many were serially examined, comparison with his data is limited. On the other hand, these values exceed those recorded by Michaëlsson (1959), which may be partly ascribed to his use of a much smaller chest electrode.

$R / S$ Ratio. On the first day of life, median values were greater in Group I than in Group II infants in leads V2 and V3 $(\mathrm{p}<0.05)$. The ratio tended to be more than 1 in right and less than 1 
TABLE III

S Wave Amplitude in 1/10 mV showing Minimum, Mean, Maximum, and Standard Deviation DURING FIRST WEEK OF LIFE

\begin{tabular}{|c|c|c|c|c|c|c|c|c|c|c|c|c|c|}
\hline \multirow{2}{*}{ Lead } & \multirow{2}{*}{ Day } & \multicolumn{6}{|c|}{ Group I } & \multicolumn{6}{|c|}{ Group II } \\
\hline & & No. & $\begin{array}{c}\text { No. } \\
\text { zeros }\end{array}$ & Min. & Mean & Max. & S.D. & No. & $\begin{array}{c}\text { No. } \\
\text { zeros }\end{array}$ & Min. & Mean & Max. & S.D. \\
\hline \multirow{4}{*}{ V4R } & 1 & 38 & 18 & $0 \cdot 5$ & $1 \cdot 4$ & $8 \cdot 0$ & $2 \cdot 1$ & 28 & 8 & $0 \cdot 5$ & 1.9 & $\begin{array}{r}(14.0) \\
7.0 \\
(8.0)\end{array}$ & $2 \cdot 1$ \\
\hline & 2 & 38 & 7 & 0.5 & $2 \cdot 1$ & $6 \cdot 0$ & $1 \cdot 8$ & 29 & 10 & $0 \cdot 5$ & 1.9 & $\begin{array}{r}6.0 \\
(11.5)\end{array}$ & 1.9 \\
\hline & 3 & 38 & 8 & $0 \cdot 5$ & $1 \cdot 8$ & $7 \cdot 0$ & $1 \cdot 5$ & 30 & 8 & 0.5 & $1 \cdot 8$ & $8 \cdot 5$ & $2 \cdot 0$ \\
\hline & $5-6$ & 38 & 18 & $0 \cdot 5$ & 0.9 & $6 \cdot 0$ & $1 \cdot 4$ & 30 & 16 & 0.5 & 0.9 & 3.5 & $1 \cdot 1$ \\
\hline \multirow{3}{*}{ V1 } & $\begin{array}{l}1 \\
2\end{array}$ & $\begin{array}{l}38 \\
37\end{array}$ & $\begin{array}{l}1 \\
0\end{array}$ & $\begin{array}{l}0.5 \\
0.5\end{array}$ & $\begin{array}{l}14 \cdot 8 \\
18 \cdot 1\end{array}$ & $\begin{array}{l}36 \cdot 0 \\
39 \cdot 0\end{array}$ & $\begin{array}{l}9 \cdot 5 \\
8.5\end{array}$ & $\begin{array}{l}29 \\
30\end{array}$ & $\begin{array}{l}0 \\
1\end{array}$ & $\begin{array}{l}2.5 \\
0.5\end{array}$ & $\begin{array}{l}14 \cdot 8 \\
16.9\end{array}$ & $\begin{array}{c}(34.0) \\
26.0 \\
38.0 \\
(35.0\end{array}$ & $\begin{array}{l}6 \cdot 0 \\
8 \cdot 5\end{array}$ \\
\hline & 3 & 38 & 0 & $2 \cdot 0$ & $17 \cdot 2$ & $38 \cdot 0$ & $8 \cdot 3$ & 30 & 0 & $2 \cdot 0$ & $16 \cdot 5$ & 31.5 & $7 \cdot 4$ \\
\hline & $5-6$ & 38 & 0 & 0.5 & $11 \cdot 1$ & $27 \cdot 0$ & $7 \cdot 1$ & 30 & 0 & $1 \cdot 5$ & $11 \cdot 7$ & $22 \cdot 0$ & $4 \cdot 7$ \\
\hline \multirow{4}{*}{ V2 } & $\begin{array}{l}1 \\
2\end{array}$ & $\begin{array}{l}38 \\
38\end{array}$ & $\begin{array}{l}1 \\
0\end{array}$ & $\begin{array}{l}5 \cdot 0 \\
8.5\end{array}$ & $\begin{array}{l}20 \cdot 6 \\
23 \cdot 7\end{array}$ & $\begin{array}{l}37 \cdot 0 \\
44 \cdot 0\end{array}$ & $\begin{array}{l}8 \cdot 8 \\
8 \cdot 1\end{array}$ & $\begin{array}{l}29 \\
29\end{array}$ & $\begin{array}{l}0 \\
0\end{array}$ & $\begin{array}{l}2 \cdot 0 \\
3.0\end{array}$ & $\begin{array}{l}21 \cdot 6 \\
22 \cdot 3\end{array}$ & $\begin{array}{l}39.0 \\
38.5\end{array}$ & $\begin{array}{l}8 \cdot 6 \\
8 \cdot 0\end{array}$ \\
\hline & 3 & 38 & 0 & 9.5 & 21.0 & 39.0 & $7 \cdot 7$ & 30 & 0 & 600 & 21.7 & $(42 \cdot 0)$ & 67 \\
\hline & J & 30 & 0 & 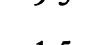 & 21 & & & & & $(6 \cdot 5)$ & & $(34 \cdot 5)$ & \\
\hline & $5-6$ & 38 & 0 & 1.5 & $15 \cdot 0$ & $34 \cdot 0$ & $6 \cdot 8$ & 30 & 0 & $7 \cdot 0$ & $16 \cdot 4$ & $23 \cdot 5$ & $4 \cdot 0$ \\
\hline \multirow[t]{2}{*}{ V3 } & $\begin{array}{l}1 \\
2 \\
3\end{array}$ & $\begin{array}{l}38 \\
37 \\
38\end{array}$ & $\begin{array}{l}0 \\
0 \\
0\end{array}$ & $\begin{array}{l}(9.0) \\
10.5 \\
10.0 \\
11.5\end{array}$ & $\begin{array}{l}23 \cdot 5 \\
26 \cdot 7 \\
25 \cdot 1\end{array}$ & $\begin{array}{l}40 \cdot 5 \\
41 \cdot 5 \\
40 \cdot 0\end{array}$ & $\begin{array}{l}7 \cdot 6 \\
8.0 \\
7 \cdot 0\end{array}$ & $\begin{array}{l}29 \\
30 \\
30\end{array}$ & $\begin{array}{l}0 \\
0 \\
0\end{array}$ & $\begin{array}{c}7.0 \\
12.0 \\
12.0 \\
(6.5)\end{array}$ & $\begin{array}{l}22 \cdot 7 \\
25 \cdot 7 \\
25 \cdot 0\end{array}$ & $\begin{array}{c}(36 \cdot 5) \\
34 \cdot 0 \\
35 \cdot 0 \\
36 \cdot 0 \\
(35 \cdot 0)\end{array}$ & $\begin{array}{l}6.0 \\
5.4 \\
5.9\end{array}$ \\
\hline & $5-6$ & 38 & 0 & $6 \cdot 0$ & $16 \cdot 8$ & $29 \cdot 0$ & $6 \cdot 1$ & 30 & 0 & 11.0 & $17 \cdot 2$ & $27 \cdot 5$ & $4 \cdot 0$ \\
\hline \multirow{3}{*}{ V4 } & 1 & 38 & 0 & $\begin{array}{c}(6 \cdot 0) \\
11 \cdot 5 \\
(10 \cdot 0)\end{array}$ & $24 \cdot 3$ & $44 \cdot 0$ & 6.5 & 29 & 0 & $8 \cdot 0$ & $21 \cdot 7$ & $\begin{array}{c}(32 \cdot 0) \\
30 \cdot 5\end{array}$ & $5 \cdot 5$ \\
\hline & $\begin{array}{l}2 \\
3\end{array}$ & $\begin{array}{l}38 \\
38\end{array}$ & $\begin{array}{l}0 \\
0\end{array}$ & $\begin{array}{l}10.5 \\
10.0 \\
(6.0)\end{array}$ & $\begin{array}{l}24 \cdot 2 \\
23 \cdot 2\end{array}$ & $\begin{array}{l}38 \cdot 0 \\
45 \cdot 0\end{array}$ & $\begin{array}{l}6.5 \\
7.0\end{array}$ & $\begin{array}{l}30 \\
29\end{array}$ & $\begin{array}{l}0 \\
0\end{array}$ & $\begin{array}{r}3.5 \\
12.0 \\
(9.5)\end{array}$ & $\begin{array}{l}23 \cdot 5 \\
22 \cdot 5\end{array}$ & $\begin{array}{l}35 \cdot 0 \\
36 \cdot 0\end{array}$ & $\begin{array}{l}7 \cdot 3 \\
5 \cdot 5\end{array}$ \\
\hline & $5-6$ & 38 & 0 & 8.5 & $18 \cdot 7$ & $32 \cdot 5$ & 4.9 & 30 & 0 & 11.5 & $17 \cdot 2$ & $37 \cdot 0$ & 3.9 \\
\hline \multirow{3}{*}{ V5 } & 1 & 38 & 0 & $\begin{array}{c}(1 \cdot 5) \\
3 \cdot 5 \\
(3.0)\end{array}$ & 13.9 & 29.0 & $5 \cdot 7$ & 29 & 0 & $2 \cdot 0$ & $12 \cdot 0$ & $\begin{array}{c}(22 \cdot 0) \\
20.0 \\
(32 \cdot 0)\end{array}$ & $4 \cdot 7$ \\
\hline & $\begin{array}{l}2 \\
3\end{array}$ & $\begin{array}{l}38 \\
38\end{array}$ & $\begin{array}{l}0 \\
0\end{array}$ & $\begin{array}{c}5.5 \\
6.0 \\
(1.5)\end{array}$ & $\begin{array}{l}13 \cdot 9 \\
15 \cdot 0\end{array}$ & $\begin{array}{l}27 \cdot 5 \\
34 \cdot 0\end{array}$ & $\begin{array}{l}6 \cdot 3 \\
7 \cdot 2\end{array}$ & $\begin{array}{l}30 \\
29\end{array}$ & $\begin{array}{l}0 \\
0\end{array}$ & $\begin{array}{c}0.5 \\
5.5 \\
(4.0)\end{array}$ & $\begin{array}{l}14 \cdot 3 \\
13 \cdot 8\end{array}$ & $\begin{array}{r}28.0 \\
28.0 \\
(20.0)\end{array}$ & $\begin{array}{l}5 \cdot 8 \\
5 \cdot 2\end{array}$ \\
\hline & $5-6$ & 38 & 0 & 5.0 & $11 \cdot 7$ & $30 \cdot 5$ & $4 \cdot 7$ & 30 & 0 & $4 \cdot 5$ & $10 \cdot 8$ & $18 \cdot 5$ & $3 \cdot 8$ \\
\hline \multirow{3}{*}{ V6 } & 1 & 38 & 0 & $\begin{array}{c}(0) \\
2 \cdot 5 \\
(0 \cdot 5)\end{array}$ & $10 \cdot 0$ & $29 \cdot 0$ & $5 \cdot 4$ & 29 & 2 & $\begin{array}{c}(1.0) \\
1.5\end{array}$ & $6 \cdot 9$ & $18 \cdot 0$ & 3.9 \\
\hline & $\begin{array}{l}2 \\
3\end{array}$ & $\begin{array}{l}38 \\
38\end{array}$ & $\begin{array}{l}0 \\
0\end{array}$ & $\begin{array}{c}1.0 \\
2.0 \\
(1.0)\end{array}$ & $\begin{array}{l}9.5 \\
9.7\end{array}$ & $\begin{array}{l}26 \cdot 5 \\
27 \cdot 0\end{array}$ & $\begin{array}{l}6 \cdot 4 \\
6 \cdot 1\end{array}$ & $\begin{array}{l}30 \\
29\end{array}$ & $\begin{array}{l}2 \\
0\end{array}$ & $\begin{array}{l}1.0 \\
1.5\end{array}$ & $\begin{array}{l}7 \cdot 4 \\
8 \cdot 3\end{array}$ & $\begin{array}{c}22.0 \\
24.5 \\
(26.0)\end{array}$ & $\begin{array}{l}4 \cdot 4 \\
6 \cdot 0\end{array}$ \\
\hline & $5-6$ & 38 & 0 & $2 \cdot 0$ & $9 \cdot 2$ & $29 \cdot 0$ & $5 \cdot 3$ & 30 & 0 & 1.0 & $7 \cdot 2$ & 16.0 & 3.6 \\
\hline
\end{tabular}


TABLE III (continued)

\begin{tabular}{|c|c|c|c|c|c|c|c|c|c|c|c|c|c|}
\hline \multirow{2}{*}{ Lead } & \multirow{2}{*}{ Day } & \multicolumn{6}{|c|}{ Group I } & \multicolumn{6}{|c|}{ Group II } \\
\hline & & No. & $\begin{array}{c}\text { No. } \\
\text { zeros }\end{array}$ & Min. & Mean & Max. & S.D. & No. & $\begin{array}{c}\text { No. } \\
\text { zeros }\end{array}$ & Min. & Mean & Max. & S.D. \\
\hline \multirow{3}{*}{$\mathrm{Ve}$} & 1 & 38 & 0 & $0 \cdot 5$ & $15 \cdot 1$ & $28 \cdot 5$ & $6 \cdot 1$ & 29 & 0 & $\begin{array}{c}(3 \cdot 0) \\
6.0 \\
(1.0)\end{array}$ & $14 \cdot 2$ & $\begin{array}{c}(25 \cdot 0) \\
24 \cdot 0\end{array}$ & $3 \cdot 9$ \\
\hline & $\begin{array}{l}2 \\
3\end{array}$ & $\begin{array}{l}38 \\
38\end{array}$ & $\begin{array}{l}0 \\
0\end{array}$ & $\begin{array}{l}0.5 \\
1.0\end{array}$ & $\begin{array}{l}14 \cdot 3 \\
12 \cdot 8\end{array}$ & $\begin{array}{l}33 \cdot 0 \\
31 \cdot 0\end{array}$ & $\begin{array}{l}5 \cdot 8 \\
5 \cdot 2\end{array}$ & $\begin{array}{l}29 \\
30\end{array}$ & $\begin{array}{l}0 \\
0\end{array}$ & $\begin{array}{l}2.0 \\
5.0\end{array}$ & $\begin{array}{l}13 \cdot 8 \\
12 \cdot 7\end{array}$ & $\begin{array}{l}27 \cdot 0 \\
24 \cdot 5\end{array}$ & $\begin{array}{l}6 \cdot 4 \\
5 \cdot 0\end{array}$ \\
\hline & $5-6$ & 37 & 1 & $2 \cdot 5$ & $7 \cdot 4$ & $16 \cdot 0$ & $3 \cdot 1$ & 29 & 0 & $2 \cdot 5$ & $8 \cdot 3$ & $16 \cdot 0$ & $3 \cdot 3$ \\
\hline \multirow{4}{*}{$2 \mathrm{~V} 1$} & & & & $(0 \cdot 5)$ & & & & & & & & $(26 \cdot 5)$ & \\
\hline & $\begin{array}{l}1 \\
2\end{array}$ & $\begin{array}{l}38 \\
38\end{array}$ & $\begin{array}{l}6 \\
4\end{array}$ & $\begin{array}{l}1.0 \\
1.0\end{array}$ & $\begin{array}{l}5 \cdot 5 \\
8 \cdot 4\end{array}$ & $\begin{array}{l}19 \cdot 5 \\
21 \cdot 5\end{array}$ & $\begin{array}{l}4 \cdot 8 \\
5 \cdot 7\end{array}$ & $\begin{array}{l}29 \\
30\end{array}$ & $\begin{array}{l}4 \\
3\end{array}$ & $\begin{array}{l}0.5 \\
0.5\end{array}$ & $\begin{array}{l}7 \cdot 5 \\
7 \cdot 5\end{array}$ & & $\begin{array}{l}5 \cdot 7 \\
6 \cdot 0\end{array}$ \\
\hline & 3 & 38 & 3 & $0 \cdot 5$ & $8 \cdot 1$ & $22 \cdot 5$ & $6 \cdot 5$ & 29 & 2 & $0 \cdot 5$ & $5 \cdot 9$ & $17 \cdot 0$ & $3 \cdot 7$ \\
\hline & $5-6$ & 38 & 5 & $1 \cdot 0$ & $5 \cdot 9$ & $29 \cdot 0$ & $5 \cdot 6$ & 30 & 3 & $0 \cdot 5$ & $5 \cdot 6$ & $11 \cdot 5$ & $3 \cdot 3$ \\
\hline \multirow{3}{*}{$2 \mathrm{~V} 2$} & $\begin{array}{l}1 \\
2\end{array}$ & $\begin{array}{l}38 \\
38\end{array}$ & $\begin{array}{l}2 \\
2\end{array}$ & $\begin{array}{l}0 \cdot 5 \\
1 \cdot 0\end{array}$ & $\begin{array}{r}9 \cdot 4 \\
11 \cdot 8\end{array}$ & $\begin{array}{l}25 \cdot 5 \\
33 \cdot 0\end{array}$ & $\begin{array}{l}6 \cdot 7 \\
7 \cdot 1\end{array}$ & $\begin{array}{l}29 \\
29\end{array}$ & $\begin{array}{l}2 \\
1\end{array}$ & $\begin{array}{c}(0.5) \\
1.5 \\
0.5\end{array}$ & $\begin{array}{l}10 \cdot 9 \\
11 \cdot 2\end{array}$ & $\begin{array}{l}25 \cdot 5 \\
23 \cdot 0\end{array}$ & $\begin{array}{l}6 \cdot 6 \\
5 \cdot 9\end{array}$ \\
\hline & 3 & 38 & 3 & $2 \cdot 0$ & $12 \cdot 4$ & $32 \cdot 0$ & $7 \cdot 6$ & 30 & 2 & $2 \cdot 5$ & $10 \cdot 3$ & $\begin{array}{c}18.0 \\
(22.5)\end{array}$ & $4 \cdot 9$ \\
\hline & $5-6$ & 37 & 3 & $0 \cdot 5$ & $8 \cdot 0$ & $30 \cdot 0$ & $6 \cdot 1$ & 30 & 0 & $0 \cdot 5$ & $8 \cdot 6$ & $18 \cdot 5$ & $4 \cdot 8$ \\
\hline
\end{tabular}

On first day of life higher values in left præcordial leads are found in Group I infants. Figures in parentheses indicate minimum and maximum values in infants without all four examinations. Calculation of mean includes zeros.

in left præcordial leads. Increases in the median with age occurred in almost all præcordial leads: these changes were significant in V4, V5, and Ve $(p<0.05)$ in Group I infants; and in V2 $(p<0.05)$, V3, and V5 $(p<0.01)$, and V4 and Ve $(p<0.001)$ in Group II infants. By the end of the week, this ratio tended to be more than 1 in all præcordial leads (Table IV).

The value of the $\mathrm{R} / \mathrm{S}$ ratio for diagnosis of ventricular hypertrophy in the newborn infant is still open to question. There is some discrepancy in the results of various investigations. In Datey and Bharucha's (1960) serial study of 44 newborn babies during the first week of life, the R/S ratio was more than 1 in leads V4R and V1 in all electrocardiograms. Similarly, in another study by Richman and Master (1951), who took daily electrocardiograms on some newborns during the first four days of life, this ratio was usually more than 1 in all chest leads during this period. On the other hand, in a report by Furman and Halloran (1951), 45 per cent of 20 normal newborns during the first week of life had an R/S ratio equal to or less than 1 in all præcordial leads, and an additional 5 per cent had a deep S wave in V1. Recently, Keith et al. (1961) compared the R/S ratio in lead V1 during the first 48 hours of life in various groups of newborns. They found that 18 per cent of 27 normal full-term infants, 37 per cent of 24 healthy premature infants, 46 per cent and 50 per cent of 11 full-term and 10 premature infants with respiratory distress had a ratio less than 1 . These results were interpreted as indicating that a higher percentage of infants with respiratory distress have a ratio less than 1. This, therefore, lends support to those who favour left-sided heart failure consequent on hypoxia as an explanation for the formation of hyaline membranes. However, in this study, 27 per cent of the infants on the first, 32 per cent on the second, and 36 per cent on the third day of life had a ratio less than 1 . These percentages resemble those encountered by Keith in normal prematures. In view of the reported variance in values, and until larger series have confirmed the higher incidence of low ratios in respiratory distress, caution should be exercised in relying on this ratio for diagnosis. 
TABLE IV

R/S Ratio in 1/10 mV showing Minimum, Median, and Maximum Values on First and Fifth OR SIXTH DAY OF LIFE

\begin{tabular}{|c|c|c|c|c|c|c|c|c|c|c|c|}
\hline \multirow[b]{2}{*}{ Lead } & \multirow[b]{2}{*}{ Day } & \multicolumn{5}{|c|}{ Group I } & \multicolumn{5}{|c|}{ Group II } \\
\hline & & No. & $\begin{array}{c}\text { No. } \\
\text { zeros }\end{array}$ & Min. & Median & Max. & No. & $\begin{array}{c}\text { No. } \\
\text { zeros }\end{array}$ & Min. & Median & Max. \\
\hline \multirow[t]{2}{*}{ V4R } & 1 & 38 & 18 & $1 \cdot 20$ & $17 \cdot 50$ & $18 \cdot 00$ & 28 & 8 & $1 \cdot 40$ & $5 \cdot 80$ & $32 \cdot 00$ \\
\hline & $5-6$ & 38 & 18 & $1 \cdot 30$ & $22 \cdot 00$ & $22 \cdot 00$ & 30 & 16 & $1 \cdot 40$ & $\infty$ & $16 \cdot 00$ \\
\hline \multirow[t]{2}{*}{ V1 } & 1 & 38 & 1 & 0.38 & $1 \cdot 38$ & $14 \cdot 50$ & 29 & 0 & 0.83 & $1 \cdot 31$ & $5 \cdot 20$ \\
\hline & $5-6$ & 38 & 0 & 0.44 & 1.59 & 40.00 & 30 & 0 & 0.93 & 1.45 & $10 \cdot \overline{70}$ \\
\hline \multirow[t]{2}{*}{ V2 } & 1 & 38 & 1 & 0.57 & $1 \cdot 28$ & 3.60 & 29 & 1 & 0.45 & 0.92 & $7 \cdot 75$ \\
\hline & $5-6$ & 38 & 0 & 0.25 & $1 \cdot 36$ & $11 \cdot 30$ & 29 & 0 & 0.69 & $1 \cdot 23$ & $3 \cdot 14$ \\
\hline \multirow[t]{2}{*}{ V3 } & 1 & 38 & 0 & 0.24 & 1.07 & $2 \cdot 10$ & 29 & 0 & $0 \cdot 36$ & 0.76 & $1 \cdot 70$ \\
\hline & $5-6$ & 38 & 0 & 0.32 & $1 \cdot 08$ & $2 \cdot 67$ & 30 & 0 & 0.44 & $1 \cdot 11$ & $2 \cdot 13$ \\
\hline \multirow[t]{2}{*}{ V4 } & 1 & 38 & 0 & 0.37 & $0 \cdot 76$ & $1 \cdot 70$ & 29 & 0 & 0.36 & 0.77 & $1 \cdot 70$ \\
\hline & $5-6$ & 38 & 0 & 0.46 & 1.07 & $2 \cdot 24$ & 30 & 1 & $0 \cdot 31$ & $1 \cdot 12$ & 1.92 \\
\hline \multirow[t]{2}{*}{ V5 } & 1 & 38 & 0 & $0 \cdot 30$ & 0.87 & $2 \cdot 11$ & 29 & 0 & $0 \cdot 19$ & 0.83 & $6 \cdot 00$ \\
\hline & $5-6$ & 38 & 0 & 0.50 & $1 \cdot 11$ & $2 \cdot 20$ & 30 & 0 & 0.38 & $1 \cdot 18$ & 3.90 \\
\hline \multirow[t]{2}{*}{ V6 } & 1 & 38 & 0 & 0.30 & 0.88 & $2 \cdot 20$ & 29 & 2 & 0.00 & 0.91 & $2 \cdot 36$ \\
\hline & $5-6$ & 38 & 0 & 0.07 & $1 \cdot 13$ & $3 \cdot 25$ & 30 & 0 & 0.25 & $1 \cdot 12$ & $6 \cdot 50$ \\
\hline \multirow[t]{2}{*}{$\mathrm{Ve}$} & 1 & 38 & 0 & 0.61 & 1.63 & $42 \cdot 00$ & 29 & 0 & $0 \cdot 70$ & $1 \cdot 63$ & 4.08 \\
\hline & $5-6$ & 37 & 1 & 0.78 & $2 \cdot 34$ & $8 \cdot 22$ & 29 & 0 & 0.97 & $2 \cdot 57$ & $7 \cdot 20$ \\
\hline \multirow[t]{2}{*}{ 2V1 } & 1 & 38 & 6 & $0 \cdot 84$ & $2 \cdot 63$ & $12 \cdot 00$ & 29 & 4 & 0.77 & $1 \cdot 70$ & 8.00 \\
\hline & $5-6$ & 38 & 5 & 0.41 & $1 \cdot 85$ & $11 \cdot 00$ & 30 & 3 & 0.78 & 1.66 & $11 \cdot 00$ \\
\hline \multirow[t]{2}{*}{$2 \mathrm{~V} 2$} & 1 & 38 & 2 & 0.63 & $1 \cdot 45$ & 26.00 & 29 & 2 & 0.41 & $1 \cdot 15$ & 9.00 \\
\hline & $5-6$ & 37 & 3 & 0.39 & $1 \cdot 73$ & 12.00 & 30 & 0 & 0.66 & $1 \cdot 14$ & 22.00 \\
\hline
\end{tabular}

Statistically significant differences between the 2 groups on the first day of life are present in leads V2 and V3. From the first to the last examination significant increases in ratio were observed in V4, V5, and Ve in Group I infants and in V2 through V5 and Ve in Group II infants. Calculations of median include $\infty$.

Patterns in Right and Left Pracordial Leads. For purposes of pattern analysis, values of $4.5 \mathrm{~mm}$. or less $(0.45 \mathrm{mV})$ were classified as a small $\mathrm{q}, \mathrm{r}$, or s wave. With this method, on initial examination in lead V4R, 9 infants had an RS, 26 an Rs, and 31 an R, qRs, qR, or rsR. A similar distribution was present at the end of the week. In $\mathrm{V} 1$, only three patterns were encountered: initially, most infants had an RS, some an Rs, and only one an R, no change took place during the week. In V6, on the first day, the majority of Group Ia infants did not have an initial Q wave (10/17), while the majority of Group IIb infants did (13/16). When the groups were combined, 8 infants had a qrs or qr, 22 an rs, rS, S, RS, or Rs, and 37 a qrS, qRs, or qRS. By the end of the week, 2, 14, and 51 infants had these patterns. With this classification, therefore, the only changes were registered in V6. These small variations in appearance of complexes, especially in right præcordial leads, indicate that until further knowledge is gained of pressure-flow relations and their influence on the neonatal electrocardiogram, the value of pattern analysis in this age-group will remain limited.

\section{COMMENT}

These differences on the first day and during the first week of life have not been reported previously. This may be ascribed to failure to study infants immediately after birth and the tendency to include infants 1 week old or more in a single group. Admittedly, an inherent error is present in serial examination especially of newborns as minor changes in body position may considerably alter electrode position. Conversely, single examinations of large series of infants demonstrate wide variations in amplitudes of deflections thereby obscuring individual trends which are paramount for understanding of significant but smaller changes.

Although there are theoretical limitations in relating electrocardiographic measurements to physiological data, several investigators have demonstrated a positive correlation between electrocardiographic and hæmodynamic findings (Cosby et al., 1952; Goodwin, 1952). Electrocardiographic changes appear to be determined largely by the load imposed on the ventricles. The 
interaction of numerous factors, such as the degree of anoxæmia, electrolyte balance, pulmonary vascular resistance, and time of closure of the ductus, undoubtedly influences the appearance of the neonatal electrocardiogram. The duration of the transitional period of adaptation to extrauterine life appears to vary, but the sequence of change is probably the same. An analogy may perhaps be drawn between the electrocardiographic findings in this study and data based on experimental work partly supported by a few physiological studies in the newborn baby (Dawes, Mott, and Widdicombe, 1954; Adams and Lind, 1957; Rowe and James, 1957).

At birth, the pressure in both ventricles is presumably equal. With expansion of the lungs and ligation of the cord, pulmonary vascular resistance falls and systemic resistance rises. During the first hour of life, and possibly shortly thereafter, a right-to-left shunt across the ductus occurs, but this then reverses (Eldridge, Hultgren, and Wigmore, 1955). At this time, high amplitude $R$ waves are recorded in right and especially mid-præcordial leads and deep $\mathrm{S}$ waves in leads from the left chest. In addition, some infants have $\mathrm{Q}$ waves in lead V4R as well as other right chest leads, generally considered evidence of an abnormal degree of right ventricular preponderance. Pulmonary flow increases, enhanced still further by left-to-right shunting through the ductus for as long as one week or even more. Increased venous return and decreased pressure in the inferior vena cava help to effect closure of the foramen ovale (Dawes, Mott, and Widdicombe, 1955). Significant right-to-left shunting across this opening apparently does not occur. Since pulmonary vascular resistance is still relatively elevated, partly as a result of the characteristics of the neonatal pulmonary vascular bed, flow from the ductus appears to be sufficient to maintain pulmonary hypertension for several days. During this period, right and mid-præcordial deflections are decreasing, and Q and $\mathrm{R}$ waves over the left chest are becoming larger. However, in some instances, pulmonary arterial pressure may remain high for a considerably longer period (Rowe and James, 1957). This is perhaps partly reflected by the small percentage of infants with persistent $Q$ waves in lead V4R and other right chest leads.

The rapid decreases in amplitude after the first hour of life, especially in leads reflecting right ventricular potential, suggest the need for serial examination, as shortly after birth as possible, of newborns who show evidence of cardiac or respiratory distress. Failure of these deflections to decrease may prove of future diagnostic value.

\section{SUMMARY}

Sixteen-lead electrocardiograms were taken on 97 healthy full-term infants on the first, second, third, and fifth or sixth day of life. Significantly higher amplitude R and S deflections were present in infants less than 1 hour of age, primarily in leads reflecting right ventricular potential. With age, the amplitude of deflections in right and mid-præcordial leads decreased, while the incidence of $\mathbf{Q}$ waves and amplitude of $R$ waves in left chest leads increased. $Q$ waves in V4R appear to be an uncommon but normal variant at this age. A positive correlation between the $\mathrm{Q}-\mathrm{R}$ time and amplitude of the R wave in V1 and V6 was found. Præcordial patterns and R/S ratios were discussed. Although there are theoretical limitations in relating electrocardiographic measurements to hæmodynamic findings, there appears to be a relation between these changes and available data on circulatory adaptation after birth.

The encouragement of Professor John Lind and the technical assistance of Miss Siv Enqvist are gratefully acknowledged.

\section{REFERENCES}

Adams, F. H., and Lind, J. (1957). Physiologic studies on the cardiovascular status of normal newborn infants (with special reference to the ductus arteriosus). Pediatrics, 19, 431.

Cosby, R. S., Levinson, D. C., Zinn, W. J., Dimitroff, S. P., and Griffith, G. C. (1952). Congenital heart disease: an analysis of electrocardiographic patterns in 44 patients with elevated right ventricular pressure. Amer. Heart J., 44, 581 .

Datey, K. K., and Bharucha, P. E. (1960). Electrocardiographic changes in the first week of life. Brit. Heart J., 3D $22,423$. 
Dawes, G. S., Mott, J. C., and Widdicombe, J. G. (1954). The fotal circulation in the lamb. J. Physiol. (Lond.), 126, 563.

- (1955). Closure of the foramen ovale in newborn lambs. J. Physiol. (Lond.), 128, 384.

Eldridge, F. L., Hultgren, H. N., and Wigmore, M. E. (1955). The physiologic closure of the ductus arteriosus in the newborn infant. J. clin. Invest., 34, 987.

Furman, R. A., and Halloran, W. R. (1951). The electrocardiogram in the first two months of life. J. Pediat., 39, 307.

Goodwin, J. F. (1952). The electrocardiogram in normal children and in children with right ventricular hypertrophy. Brit. Heart J., 14, 173.

Groedel, F. M., and Miller, M. (1944). Electrocardiographic studies in the newborn. Exp. Med. Surg., 2, 110.

Keith, J. D., Rose, V., Braudo, M., and Rowe, R. D. (1961). The electrocardiogram in the respiratory distress syndrome and related cardiovascular dynamics. J. Pediat., 59, 167.

Krumbhaar, E. B., and Jenks, H. H. (1917). Electrocardiographic studies in normal infants and children. Heart, 6, 189.

Michaëlsson, M. (1959). Electrocardiographic studies in the healthy newborn. Acta paediat. (Uppsala), 48, Suppl. 117 , p. 108.

Richman, B., and Master, A. M. (1951). The unipolar chest and extremity lead electrocardiogram in children (newborn to ten years old). Amer. Heart J., 41, 687.

Rosen, I. L., and Gardberg, M. (1957). The electrocardiogram and vectorcardiogram of the normal infant. Dis. Chest., 32, 493.

Rothfeld, E. L., Wachtel, F. W., Karlen, W. S., and Bernstein, A. (1960). The evolution of the vectorcardiogram and electrocardiogram of the normal infant. Part I. The normal newborn. Amer. J. Cardiol., 5, 439.

Rowe, R. D., and James, L. S. (1957). The normal pulmonary arterial pressure during the first year of life. J. Pediat., 51, 1 .

Schaffer, A. I., Burstein, J., Mascia, A. V., Barenberg, P. L., and Stillman, N. (1950). The unipolar electrocardiogram of the newborn infant. Amer. Heart J., 39, 588.

Walsh, S. Z. (1963a). P wave duration and P-R interval during first week of life. Brit. Heart J., $25,42$.

- (1963b). Evolution of the electrocardiogram of healthy premature infants during the first year of life. Acta paediat. (Uppsala), Suppl. 145.

- (1963c). Electrocardiographic intervals during the first week of life. Amer. Heart J., 66, 36.

- (1963d). Wolff-Parkinson-White syndrome in a healthy two-hour-old infant without paroxysmal tachycardia. J. Amer. med. Ass. In the press.

Ziegler, R. F. (1951). Electrocardiographic Studies in Normal Infants and Children. Charles C. Thomas, Springfield, Illinois.

- (1956). The importance of positive $\mathrm{T}$ waves in the right precordial electrocardiogram during the first year of life. Amer. Heart J., 52, 533 . 